\title{
Outcome after knee dislocation
}

\author{
Bruce A. Levy $\cdot$ Robert G. Marx
}

Published online: 22 July 2009

(C) Springer-Verlag 2009

Knee dislocations are limb-threatening injuries and, if not treated appropriately, may result in significant functional disability. Currently, there is a paucity of high-quality outcome studies in the literature to help guide optimal treatment strategies for these complex injuries [2]. Highlevel studies are extremely difficult to achieve because of the heterogeneity of the study population and the wide variation in injury patterns. Assessing outcomes is equally difficult as a large number of knee dislocation patients sustain polytrauma and may have other ipsilateral and contralateral limb injuries.

The literature to date has focused primarily on case series and surgical technique, with very few papers comparing treatment strategies or reporting on outcomes. Even the definition of a knee dislocation, in terms of the number of ligaments ruptured, remains contested. Many authors suggest that a minimum of three ligaments must be disrupted, while others contend that two ruptured ligaments (the PCL-intact knee dislocation) still constitute a dislocated knee. Wide variations in the definition of "acute" reconstruction are also noted, and no standardized functional scoring system has been universally reported. All of these factors make interpretation of the available literature extremely difficult.

Several controversies persist regarding treatment of the dislocated knee. Surgical timing, graft selection, repair versus reconstruction of the medial and lateral structures,

B. A. Levy $(\square)$

Department of Orthopaedic Surgery, Mayo Clinic,

200 First Street SW, Rochester, MN 55905, USA

e-mail: Levy.Bruce@mayo.edu

R. G. Marx

Hospital for Special Surgery, Weill Medical College of Cornell University, 535 East 70th Street, New York, NY, USA surgical techniques, and postoperative rehabilitation remain topics of debate. We recently performed a systematic review of the literature and found evidence to support early [ $<3$ weeks] operative intervention of all damaged ligamentous structures compared to nonoperative or delayed intervention. We also found a higher rate of revision for repair as opposed to reconstruction for the fibular collateral ligament (FCL) and posterolateral corner (PLC) [2].

In their prospective level II study [1], the authors found worse outcomes with high-energy compared to low-energy injured knees, and four ligaments compared to two or three ligament-injured knees. No change in outcomes was observed between patients that did or did not sustain neurovascular injury. In addition, they found no difference with acute ( $<2$ weeks) compared to chronic ( $>2$ weeks) surgical intervention. A high level of osteoarthritis (87\%) was also found in their series, consistent with previous reports [6].

Surgical techniques and postoperative rehabilitation protocols continue to evolve. A steep learning curve was evident in our own practice with regard to repair and/or reconstruction methods, and over time, we have become less aggressive with early range of motion. In this series, the authors went through similar changes with postoperative rehabilitation and employed numerous surgical techniques for repair and/or reconstruction, in particular for medial- and lateral-sided injuries. This indicates the lack of standardization for surgical techniques and the need for better evidence.

One of, if not the most important points to highlight is the issue of vascular assessment. We are aware of cases in which an unrecognized complete popliteal artery thrombosis occurred after a knee dislocation. At the time of presentation, radiographs were unremarkable, and the patients had a palpable dorsalis pedis pulse. No further 
diagnostic imaging was performed. The patients developed an ischemic compartment syndrome, which ultimately led to amputation. First, this underscores the importance of recognizing that the patient had sustained a knee dislocation and secondly that palpable pulses are possible in the presence of a complete arterial occlusion due to collateral flow. In this series, the authors conclude that physical examination alone is safe for the detection of vascular injury requiring repair which is consistent with a previous report by Stannard et al. [5]. However, they also state that all knee dislocation patients presenting to their institution since 2005 are now assessed with Ankle Brachial Indices (ABI), with an ABI of less than 0.8 necessitating further work up with angiogram. Mills et al. [3] showed that an $\mathrm{ABI}<0.9$ is highly predictive of major vascular injury, so we caution the reader that 0.9 should be used as a cut off, not 0.8 . When indicated, we currently use CT-angiogram (CTA) as opposed to conventional angiogram as CTA has been shown to be highly sensitive and specific; it uses the anticubital fossa as opposed to the groin for injection site, and uses one-fourth the radiation [4].

This prospective study is the largest series of knee dislocations reported to date and is an excellent contribution to the literature. The authors reported clinical and radiographic examination, outcome assessment and functional testing. In fact, this is the first study that we are aware of that has reported on functional single leg hop tests after multiligament knee reconstruction.
These highly complex injuries are not easily amenable to randomized clinical trials for many reasons. Multicenter prospective databases may be the key to future research efforts to strive toward improved patient outcomes.

\section{References}

1. Engebretsen L, Risberg MA, Robertson B, Ludvigsen TC, Johansen S (2009) Outcome after knee dislocations: a 2-9 years follow-up of 85 consecutive patients. Knee. doi:10.1007/s00167009-0869-y

2. Levy BA, Dajani KA, Whelan DB, Stannard JP, Fanelli GC, Stuart MJ, Boyd JL, MacDonald PA, Marx RG (2009) Decision making in the multiligament-injured knee: an evidence-based systematic review. Arthroscopy 25:430-438

3. Mills WJ, Barei DP, McNair P (2004) The value of the anklebrachial index for diagnosing arterial injury after knee dislocation: a prospective study. J Trauma 56:1261-1265

4. Redmond JM, Levy BA, Dajani KA et al (2008) Detecting vascular injury in lower-extremity orthopedic trauma: the role of CT angiography. Orthopedics 31:761-767

5. Stannard JP, Sheils TM, Lopez-Ben RR, McGwin G, Robinson JT, Volgas DA (2004) Vascular injuries in knee dislocation: the role of physical examination in determining the need for arteriography. J Bone Joint Surg Am 86:910-915

6. Werier J, Keating JF, Meek RN (1998) Complete dislocation of the knee-the long-term results of ligamentous reconstruction. Knee 5:255-260 